\title{
Profundidad del Músculo Masetero Medido Mediante Ultrasonido, según Índice Facial en Relación al Sexo
}

\author{
Masseter Muscle Deep Measured by Ultrasound per Facial Index Related to Sex
}

"Arnoldo Hernández Caldera; ${ }^{* *}$ Raúl Frugone Zambra; ${ }^{* * *}$ Hernán Valenzuela P. \& ${ }^{* * *}$ Víctor Retamal V.

HERNANDEZ, C. A; FRUGONE, Z. R; VALENZUELA, P. H. \& RETAMAL, V. V. Profundidad del musculo masetero medido mediante ultrasonido, según indice facial en relacion al sexo. Int. J. Morphol., 30(3):964-969, 2012.

RESUMEN: Para establecer las dimensiones en profundidad del músculo masetero según índice facial en relación al sexo, se realizó un estudio analítico no experimental entre pacientes seleccionados que acudieron al servicio de imagenología del hospital base de Linares, Chile. La muestra quedó determinada por 180 músculos maseteros, 90 de mujeres y 90 de hombres. Se clasificaron según su índice facial en euriprosopos, leptoprosopos y mesoprosopos quedando la muestra conformada por 60 músculos de individuos euriprosopos, mesoprosopos y leptoprosopos, respectivamente. Se realizó la medición de la profundidad de cada uno de los músculos maseteros utilizando para ello un examen imagenológico ecográfico. La muestra se sometió al análisis estadístico ANOVA. La profundidad del músculo masetero varió dependiendo del índice facial y en ambos sexos $(\mathrm{p}<0,05)$. Los individuos euriprosopos presentaron músculos maseteros con profundidades promedio de $11,5 \pm 2,08 \mathrm{~mm}$ para el sexo masculino y de $8,8 \pm 1,4 \mathrm{~mm}$ para el sexo femenino. Los individuos mesoprosopos presentaron profundidades de $11,4 \pm 1,6 \mathrm{~mm}$ para el sexo masculino y 7,8 $\pm 1,6 \mathrm{~mm}$ para el sexo femenino. Mientras que los individuos leptoprosopos presentaron profundidades promedio para el sexo masculino de $10,08 \pm 1,2 \mathrm{~mm}$ y $7,7 \pm 1,4 \mathrm{~mm}$ para los individuos de sexo femenino. La profundidad del músculo masetero varia dependiendo del índice facial en relación al sexo. Esta diferencia se encuentra entre el grupo de los individuos leptoprosopos y el grupo de los euriprosopos $(p<0,005)$. Se concluye que el índice facial de los individuos debe ser considerado en el diagnóstico de alteraciones de tamaño de la musculatura masticatoria.

PALABRAS CLAVE: Masetero; Ultrasonido; Hipertrofia muscular; Biotipo facial.

\section{INTRODUCCIÓN}

La musculatura esquelética posee múltiples y variadas funciones, entre las cuales se destacan sus funciones durante la actividad locomotora, en la mantención de la posición postural y del tono muscular en condiciones óptimas y durante la masticación y la respiración. Esto, al margen de la importancia de ella en el proceso morfogenético general (Chargé et al., 2004).

Son cuatro los pares musculares que forman el grupo de los músculos de la masticación: el masetero, el temporal, el pterigoideo medial y el pterigoideo lateral.

El músculo masetero tiene gran relevancia desde el punto de vista anatómico y funcional (morfofunción). En estudios en algunos mamíferos, entre ellos el humano, este músculo muestra capas de fascículos diversamente orientados con diferentes longitudes de las fibras y sarcómeros (Goto et al., 2001).
Las dimensiones del músculo masetero no han sido relacionadas claramente con respecto al biotipo facial. Al no estar establecido cuáles son los espesores en estado de salud de acuerdo al biotipo facial, tampoco se han establecido dichas medidas durante la enfermedad, como sería en la hipertrofia maseterina, cuyo diagnóstico clínico se basa en la palpación bilateral del músculo durante el máximo apriete dentario, lo que produce una prominencia exagerada del músculo. Por lo tanto, el diagnóstico clínico sólo se basa en la apreciación visual, lo que resulta en cierto modo subjetivo. Con el objetivo de estandarizar las dimensiones del músculo en relación al sexo, Frugone et al. (2010) midieron el músculo masetero y concluyeron que la sección transversal varía entre hombres y mujeres. El promedio en profundidad de los músculos maseteros fué mayor en hombres que en mujeres (individuos de sexo masculino $12,4 \mathrm{~mm}$ e individuos de sexo femenino $9,5 \mathrm{~mm}$ ), lo cual se podría considerar en el diagnóstico de hipertrofia maseterina.

\footnotetext{
* Programa de Anatomía y Biología del Desarrollo, Facultad de Medicina y Facultad de Odontología, Universidad de Chile, Santiago, Chile.

** Profesor de Fisiología Oral. Universidad del Desarrollo, Concepción, Chile.

**** Servicio de Radiología, Hospital Base de Linares, Chile.
} 
Por esto surge la necesidad de establecer un óptimo estándar en las mediciones del músculo masetero en estado de salud para que, de este modo, el diagnóstico de alteraciones morfológicas maseterinas sea realizado de mejor manera.

La realización de estas mediciones se ven favorecidas por nuevas y mejores tecnologías imagenológicas. Una de ellas corresponde a la ecografía que permite la realización de exámenes con gran exactitud (Bakke et al., 1992; Kiliadiris \& Kälebo, 1991).

El objetivo de este trabajo fue establecer la relación existente en la profundidad del músculo masetero según índice facial en individuos de ambos sexos entre 18 a 60 años de edad.

\section{MATERIAL Y MÉTODO}

Para contrastar la hipótesis nula "no existe diferencia significativa en la profundidad del músculo masetero entre individuos euriprosopos, mesoprosopos y leptoprosopos", se realizó un estudio analítico no experimental en sujetos de ambos sexos en las instalaciones del Hospital Presidente Carlos Ibáñez del Campo de la ciudad de Linares, Chile.

La unidad de muestra quedó determinada por músculos maseteros. La muestra, se seleccionó entre 90 pacientes que asistieron a efectuarse algún examen ecográfico, no odontológico, al servicio de radiología de dicho establecimiento y que cumplían con los criterios de inclusión. Fueron seleccionados los pacientes que cumplieran los siguientes criterios: Hombres y mujeres entre 18 y 60 años de edad, sin historial de trauma, con ausencia de patología muscular de tipo neoplásica, atrófica e hipertrófica que genere alteraciones en la simetría facial y con estabilidad oclusal. Se definió estabilidad oclusal a aquella relación interoclusal en la cual se encuentra al menos 4 unidades de oclusión distribuidas en forma cuadrilátera incluyéndose además las dos relaciones caninas.

El estudio se realizó siguiendo las normativas de la declaración de Helsinki y se contó con la debida autorización por escrito de los pacientes que participaron en este estudio. Quedaron seleccionados previa realización del índice facial 30 sujetos de biotipo euriprosopo, 30 sujetos de biotipo mesoprosopo y 30 sujetos de biotipo leptoprosopo tanto para el grupo de sexo femenino como para el grupo de sexo masculino. Se obtuvo así 180 músculos maseteros como unidades de muestra.
Los instrumentos de medición utilizados para la selección fueron la encuesta y la debida autorización del paciente, el examen extraoral y el examen intraoral.

A los individuos seleccionados se les midió el músculo masetero mediante examen de ultrasonido. Para ello se utilizó un equipo ecográfico Ecodopler color Toshiba Aplio 50 , ocupando un transductor de tipo lineal, con una frecuencia de $12 \mathrm{Mhz}$. Este examen fue realizado por un único médico radiólogo del hospital base de Linares, experto en ecografía entrenado para musculatura masticatoria, mediante el siguiente procedimiento:

1. Se ubicó al paciente recostado en una camilla en posición decúbito dorsal a una altura que favoreció ergonométricamente la toma del examen. El paciente se mantuvo bajo una condición de relajación muscular maseterina en cual los dientes ocluyeron suavemente $\sin$ acción de apriete.

2. Se realizo la medición ecográfica de la profundidad (sección transversal) de cada uno de los músculo masetero.

Los datos obtenidos se tabularon según el biotipo para cada unidad de la muestra. Para el análisis de los datos, se procedió a realizar el test estadístico ANOVA. De encontrar diferencias significativas al aplicar este test estadístico, se complemento con el uso de un test de Tukey, que permitirá establecer entre que grupos se expresa la diferencias estadísticas encontradas en el test ANOVA.

\section{RESULTADOS}

El promedio de profundidad para el músculo masetero en los individuos de sexo femenino según índice facial fue: euriprosopos 8,8 $\mathrm{mm}$ (DS 1,4), mesoprosopos 7,8 $\mathrm{mm}$ (DS 1,6) y leptoprosopos $7,7 \mathrm{~mm}$ (DS 1,4). Para los individuos de sexo masculino fue: euriprosopos 11,5 mm (DS 2,08), mesoprosopos 11,04 mm (DS 1,6) y leptoprosopos 10,08 $\mathrm{mm}$ (DS 1,2).

La Figura 1 muestra la distribución, en cuartiles, de los valores de la profundidad del músculo masetero en los músculos de individuos clasificados según el índice facial en relación al sexo. El análisis estadístico ANOVA para la profundidad del músculo masetero según el índice facial en relación al sexo femenino arrojó un $\mathrm{p}=0,012$, mientras que para los individuos de sexo masculino el test estadístico ANOVA arrojó un $\mathrm{p}=0,006$.

Posterior al análisis estadístico ANOVA, se sometió 

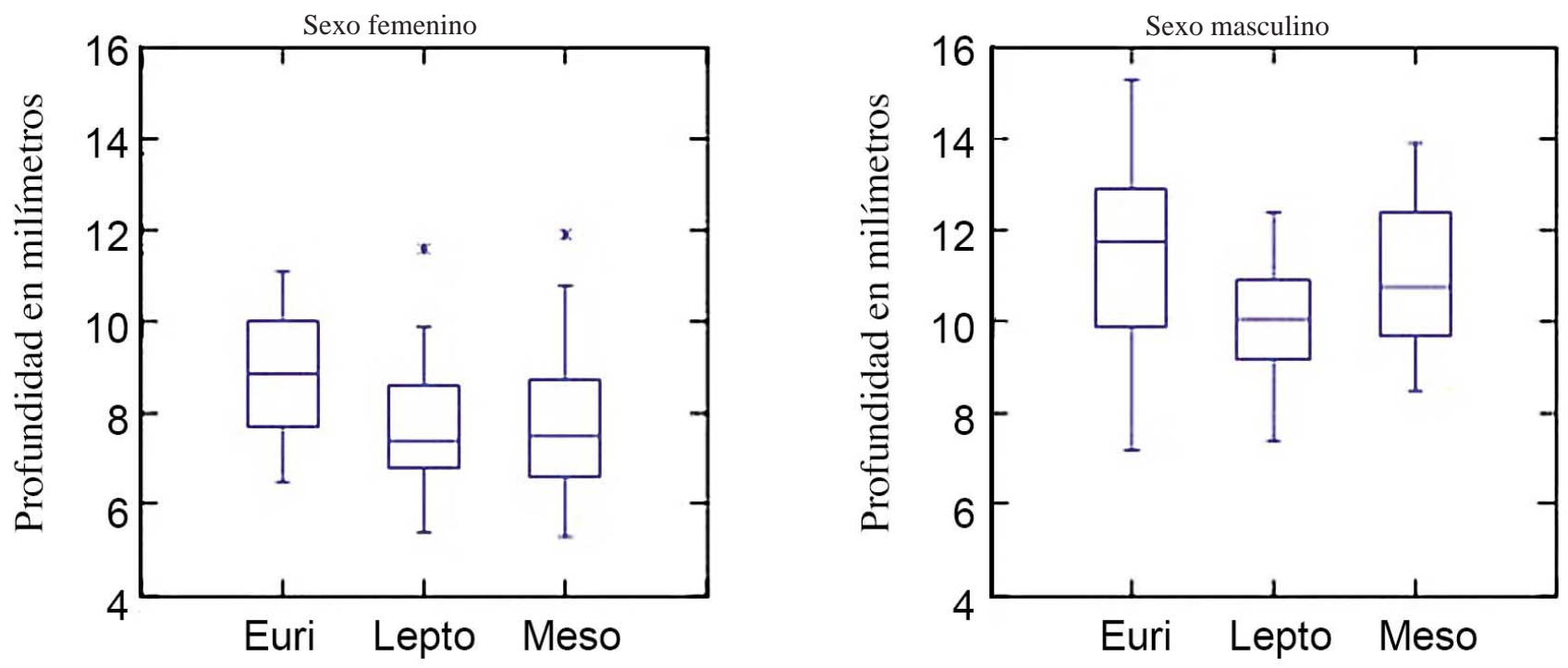

Fig. 1. Profundidad del músculo masetero, en músculos de individuos clasificados según el índice facial en relación al sexo.

la muestra a un test estadístico de Tukey para establecer entre que grupos de configuración facial se producen las diferencias en relación al sexo. Los resultados obtenidos mediante este test estadístico se observan en las Tablas I y II.

Tabla I. Matriz de probabilidad de la profundidad del músculo masetero en músculos de individuos clasificados según índice facial en individuos de sexo masculino.

\begin{tabular}{lccc}
\hline & Euriprosopo & Mesoprosopo & Leptoprosopo \\
\hline Euriprosopo & 1 & & \\
Mesoprosopo & 0,553 & 1 & \\
Leptoprosopo & 0,005 & 0,081 & 1 \\
\hline $\mathrm{p}<0,05$ & & &
\end{tabular}

Tabla II. Matriz de probabilidad de la profundidad del músculo masetero en músculos de individuos clasificados según índice facial en individuos de sexo femenino.

\begin{tabular}{lccc}
\hline & Euriprosopo & Mesoprosopo & Leptoprosopo \\
\hline Euriprosopo & 1 & & \\
Mesoprosopo & 0,033 & 1 & \\
Leptoprosopo & 0,02 & 0,981 & 1 \\
\hline $\mathrm{p}<0,05$ & & &
\end{tabular}

\section{DISCUSIÓN}

La información existente con respecto a la relación entre tipos faciales y dimensiones del músculo masetero no se han establecido claramente. Estudios previos han demostrado que sólo la profundidad del músculo varía entre individuos de distinto sexo.
La variables etáreas se debieron controlar ya que se ha establecido que existe una importante reducción de la profundidad maseterina a medida que la edad de los individuos aumenta (Bakke et al.; Kiliadiris \& Kälebo; Newton et al., 1993). Por esta razón, en este estudio sólo se evaluaron individuos entre 18 y 60 años con un promedio de edad de 35,9 años.

Del mismo modo que fueron controladas las variables etáreas, también lo fueron, pero en forma parcial, las variables funcionales ya que sólo se estudiaron a aquellos individuos con estabilidad oclusal, según la definición operacional. La profundidad del músculo masetero se relacionaría de modo positivo y significativo de acuerdo al número de contactos oclusales y por ende a la fuerza de masticación (Kasai et al., 1994). Esta es la razón por la cual, en parte, se podría explicar la variabilidad de las mediciones.

La ultrasonografía permite una buena definición de dibujo del músculo lo que ha permitido utilizar este método imagenológico como instrumento para medir el grosor de la porción superficial del músculo masetero con una buena reproducibilidad y exactitud (Bakke et al.; Kiliadiris \& Kälebo).

En este estudio se pudo establecer que las diferencias en cuanto a la morfología del músculo masetero y el biotipo facial se establecen en la dimensión transversal del músculo (profundidad) y específicamente entre los grupos euriprosopo y leptoprosopo. Resultados similares obtuvie- 
ron Kiliaridis \& Kälebo, quienes establecieron que los individuos que poseen músculos maseteros delgados tienen una cara más larga en proporción a su ancho facial, situación que se hace más obvia cuando el grosor del músculo masetero se relaciona con la proporción obtenida entre altura facial y ancho intergonial. Esto se explica por el hecho que la medición del ancho intergonial incluye también, y en forma parcial, a la medición del grosor maseterino (Kiliaridis \& Kälebo). Es por esto, que la correlación existente entre grosor maseterino y el índice entre la altura facial y el ancho intergonial es mucho más fuerte en comparación a la correlación existente entre el ancho del músculo masetero y el índice obtenido entre la altura facial y el ancho bicigomático (Kiliaridis \& Kälebo).

Weis \& Hillen (1984) también determinaron que individuos braquicefálicos y euriprosopos presentaban un grosor maseterino mayor en comparación a otros individuos. Resultados semejantes fueron obtenidos por Hannam \& Wood (1989), los que reportaron una correlación positiva entre el grosor maseterino y el ancho bicigomático.

Los valores obtenidos de las distintas dimensiones del músculo masetero vinieron a confirmar la gran variabilidad de éstos, situación que había sido descrita previamente en la literatura (Bakke et al.; Kiliaridis \& Kälebo; Goto et al.; Van Eijden et al., 1997). Kiliaridis \& Kälebo determinaron que los individuos de sexo masculino presentan una profundidad del músculo masetero en promedio de $9,7 \mathrm{~mm}$, mientras que los individuos de sexo femenino presentan una profundidad en promedio de 8,7 $\mathrm{mm}$. En el presente estudio los valores promedios para estas mismas variables fueron levemente superior en los individuos de sexo masculino, y levemente inferior en los individuos de sexo femenino. Estas diferencias en los resultados con respecto a la variabilidad de los valores de la profundidad del músculo masetero, fueron también observadas en otros estudios de tipo imagenológicos (Bakke et al.; Kiliaridis \& Kälebo; Goto et al.; Van Eijden et al.).

Frugone et al. establecieron que existen diferencias significativas entre los músculos maseteros de individuos de ambos sexos ( $\mathrm{p}=0,0005)$, teniendo los músculos de los hombres mayor profundidad, lo cual se explica por el hecho que existe una diferencia de tamaño corporal entre ambos sexos. Este resultado concuerda con el resultado obtenido por Kiliaridis \& Kälebo.

$\mathrm{Al}$ analizar los resultados, se establece que en el caso de la profundidad del músculo masetero según el índice facial en relación al sexo existen diferencias significativas tanto en el grupo de músculos de individuos de sexo femenino $(\mathrm{p}=0,012)$ como en el de sexo masculino $(\mathrm{p}=0,006)$. Los resultados indican que tanto los hombres como las mujeres presentan una profundidad del músculo masetero que se relaciona directamente con el tipo facial que ellos presenten.

Kiliaridis \& Kälebo establecieron que los individuos con un patrón facial de cara larga tienen músculos maseteros con una menor profundidad, situación que sólo fue evidenciada en individuos de sexo femenino. Mientras que Weis \& Hillen al igual que Hannam \& Wood establecieron que individuos de cara ancha presentan músculos maseteros con una mayor profundidad. Los resultados obtenidos concuerdan con los vistos en la literatura, existiendo sólo discordancia con los resultados obtenidos por Kiliaridis \& Kälebo, ya que en el presente estudio las diferencias significativas se en los individuos de ambos sexos. Esto último se explica por el hecho que en el estudio de Kiliaridis \& Kälebo solamente se evaluaron 20 músculos y además si se considera que existen individuos que poseen profundidades del músculo masetero con valores semejantes a pesar de ser clasificados como de patrones faciales distintos, explica en cierto sentido la no obtención de diferencias significativas al analizar a los individuos de sexo masculino. Posiblemente los individuos de sexo femenino participantes en el estudio de Kiliaridis \& Kälebo presentaban un patrón facial extremo, determinando que las diferencias fueran significativas.

Los resultados obtenidos para la profundidad del músculo masetero en hombres, determinaron que existe una diferencia significativa entre los músculos de individuos euriprosopos y los músculos de individuos leptoprosopos $(\mathrm{p}=0,005)$, en tanto que también existieron diferencias pero no significativas entre los músculos de individuos euriprosopos y mesoprosopos; y entre los músculos de individuos mesoprosopos y leptoprosopos. Esto se puede explicar por el hecho que existe una mayor diferencia en la profundidad de los músculos entre los tipos faciales más extremos. En los músculos de individuos de sexo femenino existieron diferencias significativas no solamente entre los músculos de individuos euriprosopos y leptoprosopos $(\mathrm{p}=0,02)$, sino también entre los músculos de individuos euriprosopos y mesoprosopos ( $\mathrm{p}=0,03$ ) (Tabla III).

En ambos sexos existen diferencias significativas entre los músculos de individuos euriprosopos y leptoprosopos, es en los individuos de sexo masculino donde la diferencia se hace más significativa. Por lo tanto, en este grupo de individuos es donde queda mejor demostrada la posible influencia de la morfofunción maseterina sobre la morfología facial.

La relación existente entre el grosor muscular maseterino y la fuerza masticatoria como también con respecto a la actividad electromiográfica ha sido ampliamente 
reconocida, indicando que existe una relación positiva entre las variables anteriormente nombradas (Bakke et al.; Weis \& Hillen; Ingervall \& Helkimo, 1978). Es así que se ha estudiado las relaciones existentes entre la fuerza masticatoria desarrollada por los músculos masticatorios en relación a variadas características morfológicas faciales (Bakke et al.; Ingervall \& Helkimo; Ringqvist, 1973; Proffit et al., 1983). De este modo, se puede relacionar de forma indirecta el grosor maseterino con los parámetros morfológicos faciales (Bakke et al.; Ingervall \& Helkimo; Ringqvist; Proffit et $a l$.). Estudios de este tipo han concluido que aquellos individuos que presentan pequeños valores de fuerza masticatoria máxima poseen caras largas y ángulos mandibulares obtusos, mientras que aquellos individuos que poseen valores elevados de fuerza masticatoria máxima, presentan una inclinación anterior de la mandíbula, tendencia al paralelismo entre las bases maxilares, altura facial anterior pequeña y un ángulo mandibular pequeño (Bakke et al.; Ingervall \& Helkimo; Ringqvist; Proffit et al.; Kasai et al.). Los resultados de estos estudios coincidieron con los obtenidos por Bakke et al., cuando las mediciones fueron realizadas en máximo apriete dentario, mientras que al realizar las mediciones en un estado de relajación muscular las correlaciones no fueron significativas con respecto a los parámetros morfológicos faciales. Otro estudio pero esta vez utilizando las propiedades de la tomografía computada, permitió obtener el volumen aproximado del músculo masetero, con el objetivo de encontrar alguna relación con los parámetros morfológicos faciales, estableciéndose que los individuos con grandes volúmenes maseterinos presentan una inclinación anterior de la mandíbula y un ángulo mandibular pequeño (Gionhaku \& Lowe, 1989).

La disminución de la eficiencia de los músculos elevadores mandibulares se ha asociado con la etiología de la morfología de cara larga, postulándose el concepto de cadenas musculares posteriores verticales (Sassouni, 1969). Este concepto señala que existe un grupo de músculos constituidos por el temporal, masetero y pterigoideo medial, que tienen como función controlar el crecimiento vertical del cráneo, pensándose que este grupo de músculos en los individuos de cara larga se orientan oblicuamente y en una posición posterior con respecto a la articulación temporomandibular (Sassouni). Estudios posteriores han concluido que la orientación del músculo masetero no influye ni se relaciona con la altura facial (van Spronsen et al., 1997). Con respecto a esto último, se determinó que la altura facial anterior no se correlaciona con los músculos elevadores mandibulares, sino por el contrario, la correlación se establece con los músculos depresores mandibulares, más específicamente con respecto al músculo pterigoideo lateral y al vientre anterior del músculo digástrico (van Spronsen et al.).
Al ser la profundidad una dimensión fácil de evaluar al examen facial, y ser una de las dimensiones que se ve en mayor grado afectada durante trastornos de crecimiento muscular, y ante los resultados obtenidos en este estudio, se establece que al evaluar a esta dimensión en conjunto con la realización del análisis facial frontal, se puede tener una referencia para poder establecer cuándo este músculo se encuentra morfológicamente normal de acuerdo a las características faciales de los individuos o cuándo este músculo se encuentra aumentado o disminuido de tamaño.

Se debe recordar lo que Moss estableció: los huesos y cartílagos carecen de la propiedad para determinar su crecimiento, por lo tanto éste es una respuesta al crecimiento intrínseco de tejidos asociados (Enlow, 1992). De este modo, el código genético para el crecimiento esquelético cráneo-facial se encontraría fuera del esqueleto óseo, dando origen al termino "matriz funcional", que se refiere a la totalidad de los tejidos asociados a una sola función, siendo ésta la que permite originar y mantener a la unidad esquelética (Enlow). Es así que se puede establecer que factores como el crecimiento muscular, la migración e inserción de los músculos, las variaciones en la función neuromuscular y la función anormal afectan de manera notable algunas características de la forma y del crecimiento cráneo-facial (Enlow). Por este motivo es que un correcto ajuste de las inserciones de los músculos en el tejido óseo determina en gran medida un correcto crecimiento del cráneo (Enlow; Sato et al., 1994).

En el músculo masetero y de acuerdo a lo expresado tanto en los aspectos teóricos como en los resultados obtenidos en este estudio, se puede establecer que la morfofunción del músculo influye en la morfología facial. Considerando que la profundidad es la única dimensión del músculo masetero que se relaciona con el patrón facial, se podría establecer que el crecimiento que tenga este músculo en sentido transversal, debido a las influencias genéticas y ambientales, durante la etapa de configuración facial, y a expensas de un aumento del número de células musculares, influirá en la determinación del patrón facial en el adulto en conjunto con otros factores, sean genéticos o funcionales.

Se concluye que el biotipo facial se relaciona con la sección transversal del músculo masetero en ambos sexos. Esta relación se establece fundamentalmente entre los biotipos faciales extremos, vale decir entre individuos euriprosopos y leptoprosopos. Los valores de la profundidad del músculo según sexo deben ser considerados en conjunto con el análisis del biotipo facial durante el examen extraoral con el objetivo de poder realizar diagnósticos más certeros, como el diagnóstico de hipertrofia maseterina. 
HERNANDEZ, C. A; FRUGONE, Z. R; VALENZUELA, P. H; RETAMAL, V.V. Masseter muscle deep measured by ultrasound per facial index related to sex. Int. J. Morphol., 30(3):964-969, 2012.

SUMMARY: To establish the dimensions of the masseter muscle deep as a facial index in relation to gender, an analytical study was conducted among patients who were attended at the imaging service Linares Hospital, Chile. The sample was 180 masseter muscles, 90 of them belonging to female individuals and 90 individuals belonging to male subjects. The subjects were classified according to facial index in euryprosopous, mesoprosopous and leptoprosopous. Each group was composed of 60 muscles. The depth of each masseter muscles was performing using an ultrasound-imaging test. The sample was subjected to ANOVA statistical analysis. The depth of masseter muscles varied depending facial index in both sexes ( $\mathrm{p}<0.05$ ). Euryprosopous individuals showed an average of $11.5 \pm 2.08 \mathrm{~mm}$ for male and $8.8 \pm 1.4 \mathrm{~mm}$ for female. Mesoprosopous individuals showed an average of $11.4 \pm 1.6 \mathrm{~mm}$. for male and $7.8 \pm 1.6 \mathrm{~mm}$ for female. Leptoprosopous individuals showed an average of $10.08 \pm 1.2 \mathrm{~mm}$ for male and $7.7 \pm 1.4 \mathrm{~mm}$. for female. The depth of the masseter muscle varies depending of facial index on both sexes. The difference is significant between muscles of leptoprosopous and euryprosopous subjects ( $p<0.005$ ). It was concluded that the facial index of individuals should be considered in the diagnosis of altered masticatory muscle size.

KEY WORDS: Masseter muscle; Ultrasound; Muscular hypertrophy; Facial biotype.

\section{REFERENCIAS BIBLIOGRÁFICAS}

Bakke, M.; Tuxen, A.; Vilmann, P.; Jensen, B. R.; Vilmann, A. \& Toft, M. Ultrasound image of human masseter muscle related to bite force, electromyography, facial morphology, and occlusal factors. Scand. J. Dent. Res., 100(3):164-71, 1992.

Chargé ,S. B. \& Rudnicki, M. A. Cellular and molecular regulation of muscle regeneration. Physiol. Rev., 84(1):209-38, 2004.

Enlow, D. H. Crecimiento Maxilofacial. $3^{\mathrm{a}}$ ed. México, Interamericana - McGraw Hill, 1992. pp. 10-1, 290, 292-311.

Frugone, R.; Hernandez, A.; Valenzuela, H. \& Retamal, V. Dimensiones del musculo masetero medido mediante ultrasonido, según sexo. Rev. Fac. Odontol. Univ. Antioq., 22(1):7-11, 2010.

Gionhaku, N. \& Lowe, A. A. Relationship between jaw muscle volume and craniofacial form. J. Dent. Res., 68(5):805-9, 1989.

Goto, T. K.; Langenbach, G. E. \& Hannam, A. G. Length changes in the human masseter muscle after jaw movement. Anat. Rec., 262(3):293-300, 2001.

Hannam, A. G. \& Wood, W. W. Relationships between the size and spatial morphology of human masseter and medial pterygoid muscles, the craniofacial skeleton, and jaw biomechanics. Am. J. Phys. Anthropol., 80(4):429-45, 1989.

Ingervall, B. \& Helkimo, E. Masticatory muscle force and facial morphology in man. Arch. Oral Biol., 23(3):203-6, 1978.

Kasai, K.; Richards, L. C.; Kanazawa, E.; Ozaki, T. \& Iwasawa, T. Relationship between attachment of the superficial masseter muscle and craniofacial morphology in dentate and edentulous humans. J. Dent. Res., 73(6):1142-9, 1994.

Kiliaridis, S. \& Kälebo, P. Masseter muscle thickness measured by ultrasonography and its relation to facial morphology. J. Dent. Res., 70(9):1262-5, 1991.
Newton, J. P.; Yemm, R.; Abel, R. W. \& Menhinick, S. Changes in human jaw muscles with age and dental state. Gerodontology, 10(1):16-22, 1993.

Proffit, W. R.; Fields, H. W. \& Nixon, W. L. Occlusal forces in normal- and long-face adults. J. Dent. Res., 62(5):566-70, 1983.

Ringqvist, M. Isometric bite force and its relation to dimensions of the facial skeleton. Acta Odontol. Scand., 31(1):35-42, 1973.

Sassouni, V. A classification of skeletal facial types. Am. J. Orthod., 55(2):109-23, 1969.

Sato, I.; Ishikawa, H.; Shimada, K.; Ezure, H. \& Sato, T. Morphology and analysis of the development of the human temporomandibular joint and masticatory muscle. Acta Anat. (Basel), 149(1):55-62, 1994.

Van Eijden, T. M.; Korfage, J. A. \& Brugman, P. Architecture of the human jaw-closing and jaw-opening muscles. Anat. Rec., 248(3):464-74, 1997.

van Spronsen, P. H.; Koolstra, J. H.; van Ginkel, F. C.; Weijs, W. A.; Valk, J. \& Prahl-Andersen, B. Relationships between the orientation and moment arms of the human jaw muscles and normal craniofacial morphology. Eur. J. Orthod., 19(3):31328,1997

Weijs, W. A. \& Hillen, B. Relationships between masticatory muscle cross-section and skull shape. J. Dent. Res., 63(9):1154-7, 1984.

Dirección para correspondencia:

Dr. Arnoldo Alejandro Hernández Caldera.

Instituto de Ciencias Biomédicas

Facultad de Medicina

Universidad de Chile, Santiago

CHILE

Recibido : 02-01-2012

Aceptado: 23-06-2012

Email: ahernandezc@med.uchile.cl 